\title{
A CONVERSE TO THE MEAN VALUE PROPERTY ON HOMOGENEOUS TREES
}

\author{
MASSIMO A. PICARDELLO AND WOLFGANG WOESS
}

\begin{abstract}
The homogeneous tree $\mathbf{T}$ of degree $q+1 \quad(q \geq 2)$ may be considered as a discrete analogue of the open unit disc D. On D, every harmonic function satisfies the mean value property (MVP) at every point. Conversely, positive functions on D having the MVP with respect to a ball with specified radius at each point of $\mathbf{D}$ are harmonic under certain assumptions concerning the radius function: results of this type are due to J. R. Baxter, W. Veech and others. Here we consider harmonic functions on $\mathbf{T}$ with respect to a natural choice of a discrete Laplacian: the analogous MVP is true in this setting. We present a Lipschitz-type condition on the radius function (which now has integer values and refers to the discrete metric of $\mathbf{T}$ ) under which harmonicity holds for positive functions whose value at each point is the mean of its values over the ball of the radius assigned to this point. The method is based upon our previous results concerning the geometrical realization of Martin boundaries of certain transition operators as the space of ends of the underlying graph.
\end{abstract}

\section{INTRODUCTION}

The homogeneous tree $\mathbf{T}=\mathbf{T}_{q}$ of degree $q+1 \quad(q \geq 2)$ is the unique connected graph without circles and the property that each vertex has exactly $q+1$ neighbors; $\mathbf{T}$ is infinite. On $\mathbf{T}$, we define the discrete Laplacian by

$$
\Delta f(x)=f(x)-\frac{1}{q+1} \sum_{y \sim x} f(y),
$$

where $f$ is a real function on (the vertex set of) $\mathbf{T}$ and $\sim$ is the adjacency relation. The discrete Laplacian can be studied for any locally finite graph, see e.g. [Do, DK, Ge, Mo]. A harmonic function is a function whose image under $\Delta$ has constant value zero; $\mathscr{H}$ denotes the set of harmonic functions and $\mathscr{H}^{+}$ the cone of positive ones.

If $\rho$ is a positive integer and $x \in \mathbf{T}$ (we shall constantly write $x \in \mathbf{T}$ if $x$ is a vertex of $\mathbf{T}$ ), then the ball of radius $\rho$ centered at $x$ is

$$
B(x, \rho)=\{y \in \mathbf{T} \mid d(x, y) \leq \rho\},
$$

Received by the editors September 1, 1987.

1980 Mathematics Subject Classification (1985 Revision). Primary 31C05, 60J50; Secondary $05 \mathrm{C} 05,60 \mathrm{~J} 15$.

Key words and phrases. Homogeneous tree, harmonic functions, mean value property, Martin boundary. 
where the distance $d(x, y)$ is the minimal number of edges in a path in $\mathbf{T}$ connecting $x$ and $y$;

$$
b(\rho)=\# B(x, \rho)=\frac{q+1}{q-1} q^{\rho}-\frac{2}{q-1} .
$$

The mean value property (MVP) is the following.

Lemma 1. If $h$ is harmonic, $x \in \mathbf{T}$ and $\rho \in \mathbf{N}$ then

$$
h(x)=\frac{1}{b(\rho)} \sum_{y \in B(x, \rho)} h(y) .
$$

Proof. Consider the stochastic transition operator $P=(p(x, y))_{x, y \in \mathbf{T}}$, where

$$
p(x, y)= \begin{cases}1 /(q+1), & \text { if } x \sim y, \\ 0, & \text { otherwise }\end{cases}
$$

It operates on functions (column vectors) on $\mathbf{T}$ by left matrix multiplication, and $P^{n}=\left(p^{(n)}(x, y)\right)_{x, y \in \mathbf{T}}$ denotes its $n$th operator (matrix) power. In the same way, define $Q=(q(x, y))_{x, y \in \mathbf{T}}$ by

$$
q(x, y)= \begin{cases}1 / b(\rho), & \text { if } y \in B(x, \rho), \\ 0, & \text { otherwise. }\end{cases}
$$

Then it is well known [Ca2, FP] that there is a polynomial $\phi_{\rho}$ of degree $\rho$ such that $Q=\phi_{\rho}(P)$ and $\phi_{\rho}(1)=1$. If $h \in \mathscr{H}$, then $P h=h$ and hence $Q h=\phi_{\rho}(P) h=\phi_{\rho}(1) h=h$.

We are now in a similar situation as in classical potential theory: let $\mathbf{D}$ be an open region in Euclidean space, $x \in \mathbf{D}$ and $B(x, \rho)$ a ball of (Euclidean) radius $\rho$ centered at $x$, which is contained in $\mathbf{D}$. If $h$ is a harmonic function on $\mathbf{D}$, then its value at $x$ is the mean of its values over $B(x, \rho)$, defined with respect to Lebesgue measure. If one specifies a radius $r(x)$ at each $x$ in $\mathbf{D}$ and considers positive or bounded functions whose value at each $x$ is equal to the mean over $B(x, r(x))$, then a converse to the MVP is a statement which gives conditions on the radius function and on the boundary of $\mathbf{D}$, under which any such function has to be harmonic. Results of this type have been given by [Ke, $\mathrm{Fe}, \mathrm{He}, \mathrm{Ba} 1, \mathrm{Ba} 2, \mathrm{Ve} 1, \mathrm{Ve} 2]$. We state a theorem due to W. Veech [Ve2].

If $\mathbf{D}$ is a bounded Lipschitz domain and $|r(x)-r(y)| \leq \alpha|x-y|$, where $\alpha<1$, then the converse to the MVP holds for positive functions.

In fact, Veech [Ve2] gives a more general result, which has been put in a still more general form by J. R. Baxter [Ba2].

Considering the homogeneous tree as a discrete analogue of the unit disc, we want to give a similar result. The radius function $r(\cdot)$ now has positive integer values.

Theorem. If $|r(x)-r(y)| \leq \Phi(d(x, y))$ for all $x, y \in \mathbf{T}$, where $\boldsymbol{\Phi}: \mathbf{N}_{0} \rightarrow \mathbf{N}_{0}$ satisfies

$$
\limsup _{n \rightarrow \infty} \frac{\Phi(n)}{\log _{q} n}<\frac{2}{3}
$$


then a positive function $h$ on $\mathbf{T}$ is harmonic if and only if for every $x$ in $\mathbf{T}$

$$
h(x)=\frac{1}{b(r(x))} \sum_{y \in B(x, r(x))} h(y) .
$$

Our methods follow the spirit of [Ve1, Ve2], but things get easier in the present discrete setting. Compared with Veech's result stated above, the logarithm in our theorem is not too surprising: the discrete metric of $\mathbf{T}$ corresponds to hyperbolic rather than Euclidean metric on the disc, whereas the result of [Ve2] refers to the latter.

Our paper is organized as follows. In $\S 2$, we associate with the radius function a transition operator $P_{r}$ and show that the corresponding cone $\mathscr{H}_{r}^{+}$of positive eigenfunctions with eigenvalue one satisfies $\mathscr{H}_{r}^{+}=\mathscr{H}^{+}$if and only if the Martin boundaries $\mathscr{M}_{r}$ of $P_{r}$ and $\mathscr{M}$ of $P$ (as given by (1.4)) coincide as topological spaces (are homeomorphic). It is well known [DM, Ca1] that $\mathscr{M}=\Omega$, the space of ends of $\mathbf{T}$ (see [PW1] for notation and further results concerning trees). Now our task is to show that under suitable conditions on the radius function, $\mathscr{M}_{r}=\Omega$. Throughout this paper we assume that for some $\Phi: \mathbf{N}_{0} \rightarrow \mathbf{N}_{0}$,

$$
|r(x)-r(y)| \leq \Phi(d(x, y)) .
$$

We introduce on the set of vertices of $\mathbf{T}$ a new graph structure, associated with the radius function. Then, by gradually restricting the growth rate of $\Phi$, we show that (a) this new graph has the same ends as the tree (\$3) and (b) the result of [PW2] can be applied to show that $\mathscr{M}_{r}$ coincides with the ends of this graph $(\S \S 4,5)$. Final remarks and observations are in $\S 6$, where, in particular, we exhibit an example of a radius function which does not satisfy any condition of type (1.5) and admits a bounded function in $\mathscr{H}_{r} \backslash \mathscr{H}$.

We acknowledge fruitful discussions with Yves Derriennic, who has drawn our attention onto this problem.

\section{Preliminary observations} by

In addition to the transition operator $P$ of $(1.4)$, define $P_{r}=\left(p_{r}(x, y)\right)_{x, y \in \mathbf{T}}$

$$
p_{r}(x, y)= \begin{cases}1 / b(r(x)), & \text { if } d(x, y) \leq r(x) ; \\ 0, & \text { otherwise }\end{cases}
$$

Then the set of functions having the specified MVP is $\mathscr{H}_{r}=\left\{h \mid P_{r} h=h\right\}$, whereas $\mathscr{H}=\{h \mid P h=h\}$. By Lemma $1, \mathscr{H} \subseteq \mathscr{H}_{r}$, and we intend to prove that $\mathscr{H}_{r}^{+}=\mathscr{H}^{+}$under the condition of the Theorem. As $\mathscr{H}^{+}$contains nonconstant functions, both $P$ and $P_{r}$ are transient. Thus, associated with each of the two, there are the Martin kernels

$$
K(x, y)=\frac{G(x, y)}{G(e, y)}, \quad K_{r}(x, y)=\frac{G_{r}(x, y)}{G_{r}(e, y)},
$$

where the "root vertex" $e$ is fixed once for all, $G(x, y)=\sum_{n=0}^{\infty} p^{(n)}(x, y)$ is the Green kernel and $G_{r}(x, y)$ is defined analogously. Hence, we get two possibly 
different Martin boundaries $\mathscr{M}$ and $\mathscr{M}_{r}$ describing the extreme elements of the cones $\mathscr{H}^{+}$and $\mathscr{H}_{r}^{+}$, respectively, see [KSK] and, for notation and the particular background of the present situation, [PW1, PW2].

Let $X_{n}$ and $X_{n}^{r}, n=0,1,2, \ldots$, denote the random walks (Markov chains) with state space $\mathrm{T}$ and transition operators $P$ and $P_{r}$, respectively: they are T-valued random variables defined on a probability space $(\Lambda, \mathscr{F}, \operatorname{Pr})$, and we have $\lim X_{n}=X_{\infty}$ and $\lim X_{n}^{r}=X_{\infty}^{r}$ almost surely, where $X_{\infty}$ is an $\mathscr{M}$-valued and $X_{\infty}^{r}$ an $\mathscr{M}_{r}$-valued random variable. It is well known that $\mathscr{M}=\Omega$, the space of ends of $\mathbf{T}$, each of which is an equivalence class of one way infinite paths that can be represented by an infinite geodesic starting at $e$, see [Ca1, PW1]. Furthermore, the extension of the Martin kernel $K(x, \cdot)$ onto $\mathbf{T} \cup \Omega$ is locally constant for every $x$ in $\mathbf{T}$.

Proposition 1. If $\mathscr{M}_{r}=\mathscr{M} \quad(=\Omega$ ) topologically (by homeomorphism) then $K_{r}(\cdot, \omega) \equiv K(\cdot, \omega)$ for every $\omega$ in $\Omega$, and $\mathscr{H}_{r}^{+}=\mathscr{H}^{+}$.

Proof. Denote by $\nu$ and $\nu_{r}$ the hitting probabilities on the common boundary: if $E$ is a Borel set in $\Omega$, then

$$
\nu(E)=\operatorname{Pr}\left[X_{\infty} \in E \mid X_{0}=e\right] \text { and } \nu_{r}(E)=\operatorname{Pr}\left[X_{\infty}^{r} \in E \mid X_{0}^{r}=e\right] .
$$

Now let $\varphi \in C(\Omega)$, a continuous function. Then, the corresponding solution of the Dirichlet problem for $P$,

$$
h(x)=\int_{\Omega} K(x, \omega) \varphi(\omega) \nu(d \omega),
$$

gives the continuous extension of $\varphi$ onto $\mathbf{T}$, see [De] (where this is stated for free groups $\equiv$ odd $q$, but carries over easily, see [PW1]). As $h \in \mathscr{H} \subseteq \mathscr{H}_{r}$ is a bounded function, there exists $\psi \in L^{\infty}\left(\nu_{r}\right)$ such that

$$
h(x)=\int_{\Omega} K_{r}(x, \omega) \psi(\omega) \nu_{r}(d \omega) .
$$

By the "probabilistic Fatou theorem" [KSK, Theorem 10-43],

$$
\lim _{n \rightarrow \infty} h\left(X_{n}^{r}\right)=\psi\left(X_{\infty}^{r}\right) \quad \text { almost surely, if } X_{0}^{r}=e .
$$

On the other hand, by continuity of $h \cdot \chi_{\mathrm{T}}+\varphi \cdot \chi_{\Omega}(\chi$ denoting indicator function),

$$
\lim _{n \rightarrow \infty} h\left(X_{n}^{r}\right)=\varphi\left(X_{\infty}^{r}\right) \quad \text { almost surely, if } X_{0}^{r}=e,
$$

and we have

$$
\begin{array}{r}
\int_{\Omega} K(x, \omega) \varphi(\omega) \nu(d \omega)=h(x)=\int_{\Lambda} K_{r}\left(x, X_{\infty}^{r}\right) \psi\left(X_{\infty}^{r}\right) d \operatorname{Pr} \\
=\int_{\Lambda} K_{r}\left(x, X_{\infty}^{r}\right) \varphi\left(X_{\infty}^{r}\right) d \operatorname{Pr}=\int_{\Omega} K_{r}(x, \omega) \varphi(\omega) \nu_{r}(d \omega)
\end{array}
$$

for every $x$ in T. Setting $x=e$, we get

$$
\int_{\Omega} \varphi(\omega) \nu(d \omega)=\int_{\Omega} \varphi(\omega) \nu_{r}(d \omega)
$$


for every $\varphi \in C(\Omega)$, and $\nu=\nu_{r}$. Fixing $x \in \mathbf{T}$ and substituting $\nu$ for $\nu_{r}$ in (2.3), we see that $K(x, \cdot) \equiv K_{r}(x, \cdot) \quad \nu$-almost surely. As $\nu$ has support $\Omega$ [De, PW1] and the kernels are continuous, we obtain

$$
K(x, \omega)=K_{r}(x, \omega) \text { on } \mathbf{T} \times \Omega,
$$

and $\mathscr{H}^{+}=\mathscr{H}_{r}^{+}$.

\section{THE GRAPH ASSOCIATED WITH THE RADIUS FUNCTION}

The transition operator $P_{r}$ should not be considered a priori to be strongly linked with the tree structure: if $r(x)$ is very large for some $x \in \mathbf{T}$, then from $x$ one can reach some $y$ at large distance in a single step. Hence, with $P_{r}$, we associate another graph $\Gamma_{r}$ with the same vertex set as the tree, and the following set $\mathbf{E}=\mathbf{E}\left(\Gamma_{r}\right)$ of unoriented edges:

$$
[x, y] \in \mathbf{E} \quad \text { if and only if } d(x, y) \leq \max \{r(x), r(y)\} .
$$

In other words, $[x, y]$ is an edge of $\Gamma_{r}$ if and only if $p_{r}(x, y)>0$ or $p_{r}(y, x)>0$. Our new graph has more edges, and it contains $\mathbf{T}$ as a spanning subtree. Thus, given an end of $\mathbf{T}$, as a class of equivalent paths (see e.g. [PW2]) it is contained in an end of $\Gamma_{r}$. Hence, if $\Gamma_{r}$ is locally finite, there is a natural continuous surjection from the end compactification of the tree onto the end compactification of $\Gamma_{r}$, which extends the identity mapping on the common vertex set. In order to have equality ( $\equiv$ homeomorphism) of the two, we need the following.

Lemma 2. If lim $\sup _{n \rightarrow \infty} \Phi(n)-n<\infty$, then two different geodesics of the tree originating at e represent different ends of $\Gamma_{r}$.

Proof. (Note that $\Gamma_{r}$ is not necessarily locally finite under the above condition on $\Phi$.) Let $\pi=\left[e=x_{0}, x_{1}, x_{2}, \ldots\right], \pi^{\prime}=\left[e=x_{0}^{\prime}, x_{1}^{\prime}, x_{2}^{\prime}, \ldots\right]$ be the two geodesics and $c=x_{k}=x_{k}^{\prime}$ their last common vertex (confluent). By assumption, $\Phi(n) \leq n+R, R \in \mathbf{N}$. Now set $\rho=k+r(c)+R$. We claim that in $\Gamma_{r}$, there is no finite path outside of $B(e, \rho)$ (the latter defined with respect to the tree metric!) which connects $\pi$ and $\pi^{\prime}$. Assume the contrary. Then there are $l, m>\rho$ and $x_{l}=y_{0}, y_{1}, y_{2}, \ldots, y_{s}=x_{m}^{\prime}$ in $\mathbf{T} \backslash B(e, \rho)$ which constitute a $\Gamma_{r}$-path: $d\left(y_{i-1}, y_{i}\right) \leq \max \left\{r\left(y_{i-1}\right), r\left(y_{i}\right)\right\}$ and $d\left(y_{i}, e\right)>\rho$ for all $i$. Now choose the minimal index $j$ such that the confluent of $y_{j}$ and $\pi^{\prime}$ w.r.t. $e$ does not lie on $\pi$ (again, this refers to the tree, see Figure 1 and $\mathrm{cf}$. [PW1] for notation). Suppose that $\max \left\{r\left(y_{j-1}\right), r\left(y_{j}\right)\right\}=r\left(y_{j}\right)$. (In the other case, the argument is symmetrical.) Then

$$
\begin{aligned}
r(c)+R & <d\left(y_{j-1}, c\right)=d\left(y_{j-1}, y_{j}\right)-d\left(y_{j}, c\right) \leq r\left(y_{j}\right)-d\left(y_{j}, c\right) \\
& \leq r(c)+\Phi\left(d\left(c, y_{j}\right)\right)-d\left(y_{j}, c\right) \leq r(c)+R
\end{aligned}
$$

a contradiction. 


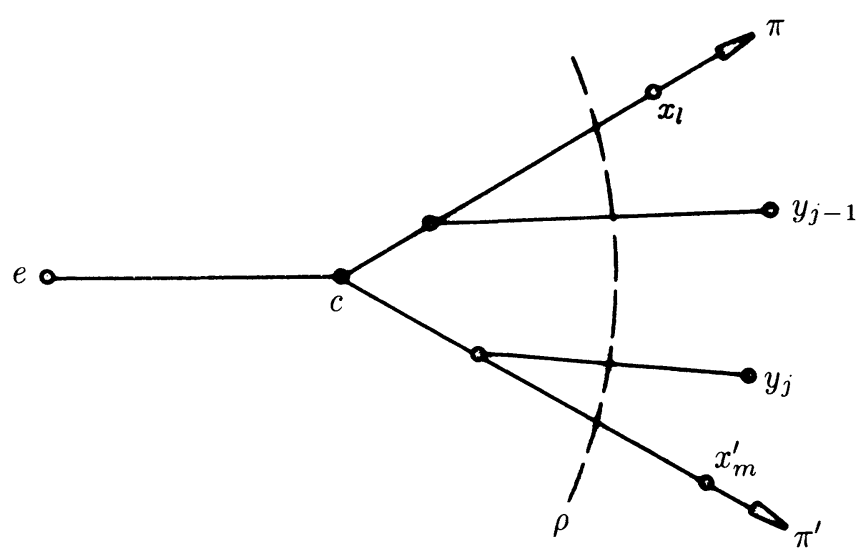

FIGURE 1

If $p_{r}(x, y)>0$, then we do not necessarily have $p_{r}(y, x)>0$. To be able to compare $\mathscr{M}_{r}$ with the space of ends of $\Gamma_{r}$, we have to meet another requirement, called uniform irreducibility in [PW2].

Lemma 3. If $\lim \sup _{n \rightarrow \infty} \Phi(n) / n<1$, then there is a positive integer $M$ with the following property: if $p_{r}(x, y)>0$ then $p_{r}^{(k)}(y, x)>0$ for some $k \leq M$. In particular, $\Gamma_{r}$ is locally finite.

Proof. By assumption, $\Phi(n) \leq A n+B$, where $0 \leq A<1$ and $B \geq 0$. Suppose $p_{r}(x, y)>0$, i.e. $d(x, y) \leq r(x)$.

Case 1. $(1-A) r(x)-B \leq 1$. Observe that $p_{r}(u, v)>0$ whenever $u \sim v$ in the tree: walking from $y$ to $x$ along the geodesic, we get $p_{r}^{(k)}(x, y)>0$ with $k=d(x, y) \leq r(x) \leq\lfloor(1+B) /(1-A)\rfloor$.

Case 2. $(1-A) r(x) ;-B>1$. If $z$ is a point on the (tree-)geodesic from $x$ to $y$, then $r(z) \geq(1-A) r(x)-B$. Jumping from $y$ to $x$ along the geodesic in steps of length at most $\lceil(1-A) r(x)-B\rceil$, we obtain $p_{r}^{(k)}(y, x)>0$, where

$$
k=\left\lceil\frac{d(x, y)}{\lceil(1-A) r(x)-B\rceil}\right\rceil \leq\left\lceil\frac{r(x)}{(1-A) r(x)-B}\right\rceil \leq\left\lceil\frac{1+B}{1-A}\right\rceil .
$$

We can choose $M=\lceil(1+B) /(1-A)\rceil$. By the above, if $y$ is a neighbor of $x$, then $p_{r}^{(k)}(x, y)>0$ for some $k \leq M$. There is only a finite number of vertices $y$ with this property, so that $x$ has only finitely many neighbors.

Corollary (see [PW2]). If lim $\sup _{n \rightarrow \infty} \Phi(n) / n<1$ then the identity mapping on $\mathbf{T}$ extends to a continuous surjection from the Martin compactification $\mathbf{T} \cup \mathscr{M}_{r}$ onto the end compactification $\mathbf{T} \cup \Omega$, which maps $\mathscr{M}_{r}$ onto $\Omega$.

\section{INTERMEDIATE SETS ALONG GEODESICS}

In this section, we assume that

$\Phi$ is nondecreasing and $\limsup _{n \rightarrow \infty} \frac{\Phi(n)}{n}<1$. 
As in the preceding section, when studying $\Gamma_{r}$, we shall always do this in terms of the geodesics and the metric of the tree. If $s \in \mathbf{T}, x \neq e$, then define $E(x)$ to be the set of all vertices $y \in \mathbf{T}$ such that $x$ lies on the geodesic from $e$ to $y$. We want to control the random walk $X_{n}^{r}$ on its way towards some end of $\Gamma_{r}$ be showing that it has to pass through certain sets which are not too big. Under assumption (4.1), the ends of $\Gamma_{r}$ can be represented by the infinite geodesics of the tree (Lemma 2), and

$$
\Phi(n) \leq n+R \quad \text { for some } R \in \mathbf{N} .
$$

Set

$$
\Psi(n)=R+\Phi(n+R) .
$$

Definition. If $x \neq e, y \in E(x)$ and $d(x, y)=r(x)+R$, then we call

$$
V(x, y)=E(x) \cap B(x, r(x)+R) \cap B(y, r(x)+\Psi(r(x)))
$$

an intermediate set.

It is easy to see that $V(x, y)=E(x) \cap B(x, r(x)+R)$ if $\Phi(r(x)+R) \geq$ $r(x)+R$, but by (4.1) this is only possible for finitely many values of $r(x)$. The following is similar to [De, Lemme 1 and PW1, Lemma 4].

Proposition 2. Let $x, y$ be as above. With probability one, the random walk $X_{n}^{r}$ has to pass through $V(x, y)$ when it is conditioned to hit $E(y) \backslash\{y\}$ starting in some point of $\mathbf{T} \backslash E(x)$.

Proof. Let $X_{0}^{r}=u \in \mathbf{T} \backslash E(x)$. Observe that we need only consider trajectories of $X_{n}^{r}, n=0,1,2, \ldots$, where $d(v, w) \leq r(v)$ for any two successive points. Assume that a given trajectory with this property, starting at $u$, hits $E(y) \backslash\{y\}$. Let $w$ be its first vertex whose confluence point $c$ with the geodesic from $x$ to $y$ (see [PW1] for notation) satisfies

$$
d(y, c)<(r(x)+\Psi(r(x))) / 2 .
$$

Observe that $c=x$ is only possible in the degenerate case where $\Phi(r(x)+R)>$ $r(x)+R$ and $V(x, y)=E(x) \cap B(x, r(x)+R)$.

If $w \in V(x, y)$, then the statement is true. So assume $w \notin V(x, y)$, and let $v$ be the preceding point on the trajectory: $d(v, w) \leq r(v)$. Assume $v \notin V(x, y)$ and let $v_{0}$ and $w_{0}$ be the points of $V(x, y)$ which are closest to $v$ and $w$, respectively. Note that $c$ lies on the geodesic from $v_{0}$ to $w_{0}$, $d\left(c, w_{0}\right)=d(c, y)$, and hence $d\left(v_{0}, w_{0}\right)=d\left(v_{0}, y\right)$. We obtain

$$
\begin{aligned}
R & \geq \Phi\left(d\left(v, v_{0}\right)\right)-d\left(v, v_{0}\right) \geq r(v)-r\left(v_{0}\right)-d\left(v, v_{0}\right) \\
& \geq d(v, w)-r\left(v_{0}\right)-d\left(v, v_{0}\right)=d\left(v_{0}, w_{0}\right)-r\left(v_{0}\right)+d\left(w_{0}, w\right) \\
& >d\left(v_{0}, y\right)-r\left(v_{0}\right) .
\end{aligned}
$$

Case 1. If $v_{0}=x$ then $d\left(v_{0}, y\right)-r\left(v_{0}\right)=R$, and the above inequality yields a contradiction. 


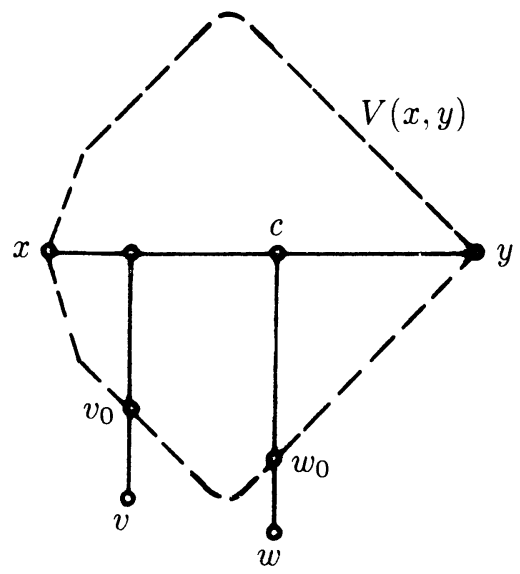

FIGURE 2

Case 2. If $v_{0} \neq x$ (see Figure 2), which is only possible in the nondegenerate case, then $d\left(v_{0}, y\right)=r(x)+\Psi(r(x)), d\left(x, v_{0}\right) \leq r(x)+R$, and, using (4.1)

$$
d\left(v_{0}, y\right)-r\left(v_{0}\right) \geq r(x)+R+\Phi(r(x)+R)-r(x)-\Phi\left(d\left(x, v_{0}\right)\right) \geq R \text {. }
$$

Again, we have arrived at a contradiction.

Note that for the tree metric, $\operatorname{diam}_{\mathbf{T}}(V(x, y))=r(x)+R+\Phi(r(x)+R)$, and $r(v) \geq \max \{1, r(x)-\Phi(r(x)+R)\}$ for $v \in V(x, y)$. Hence, in $\Gamma_{r}$,

$$
\operatorname{diam}_{\Gamma_{r}}(V(x, y)) \leq\left\lceil\frac{r(x)+R+\Phi(r(x)+R)}{\max \{1, r(x)-\Phi(r(x)+R)\}}\right\rceil,
$$

which is bounded by (4.1)

Now let $\omega \in \Omega$ be an end of $\Gamma_{r}$ (Lemma 2): $\omega$ is represented by a unique geodesic $\pi=\left[e=x_{0}, x_{1}, x_{2}, \ldots\right]$ of the tree. For $x_{i}$ on $\pi$, let $y_{i}=x_{i+\rho}$, where $\rho=r\left(x_{i}\right)+R$, and write

$$
V_{i}=V\left(x_{i}, y_{i}\right)
$$

As in [PW2], denote for $W \subset \mathbf{T}, u, v \in \mathbf{T} \backslash W$

$$
F_{W}(u, v)=\operatorname{Pr}\left[X_{n}^{r} \text { arrives at } v \text { without hitting } W \mid X_{0}^{r}=u\right] \text {. }
$$

Then, by Lemmas 2, 3 and Proposition 2, we know the following.

Lemma 4 [PW2]. If $\left\{i_{j}\right\}_{j \geq 1}$ is an increasing sequence of positive integers such that $i_{j+1}>i_{j}+r\left(x_{i_{j}}\right)+R$ and

$$
\sum_{j=1}^{\infty} \min \left\{F_{V_{i_{j+1}}}(u, v) \mid u, v \in V_{i_{j}}\right\}=\infty,
$$

then $\omega$ represents exactly one point of $\mathscr{M}_{r}$, and if this is true for all $\omega \in \Omega$, then $\mathscr{M}_{r}=\Omega$ topologically.

Note that the sets $V_{i_{j}}$ are only "one way-barriers" for $X_{n}^{r}$ moving out towards $\omega$, which is slightly weaker than the assumptions of [PW2] (where the $V_{i_{j}}$ 
should disconnect $\Gamma_{r}$ ). However, the proof of the theorem of [PW2] remains verbatim the same in the present setting.

We shall now proceed as follows: Assuming that $\Phi$ grows slowly, $i_{j}$ and $i_{j+1}$ will be kept well apart such that for each $v \in V_{i_{j}}$, there is a ball $B$ around $v$ which contains $V_{i_{j}}$ and does not intersect $V_{i_{j+1}}$. In this ball, we find "good" upper and lower bounds on the radius function. Thus, in $B$ we find a minorization of $P_{r}$ by a substochastic transition operator with constant radius. Thus, the minimum over $V_{i_{j}}$ of Lemma 4 can be minorized by the corresponding minimum of the new transition operator, which is easy to handle. Hence, our next step is to give an asymptotic evaluation of the latter when $\rho=r\left(x_{i_{j}}\right)$ tends to infinity.

\section{ASYMPTOTIC ANALYSIS AND PROOF OF THE THEOREM}

Similar as in the proof of Lemma 1, we consider a substochastic transition operator $Q_{\sigma, \tau}=Q=(q(x, y))_{x, y \in \mathrm{T}}$, where $\sigma \leq \tau$ are positive integers and

$$
q(x, y)= \begin{cases}1 / b(\tau), & \text { if } d(x, y) \leq \sigma \\ 0, & \text { otherwise }\end{cases}
$$

where $b(\tau)$ is defined as in (1.3). For the corresponding random walk $Y_{n}=$ $Y_{n}^{\sigma, \tau}, n=0,1,2, \ldots, \operatorname{Pr}\left[Y_{n+1}=y \mid Y_{n}=x\right]=q(x, y)$ and if $\sigma<\tau$, there is a positive probability at each point that $Y_{n}$ "vanishes". In our application, it will be enough to study the version $\left\{Y_{n}^{*}\right\}$ of $\left\{Y_{n}\right\}$ which is restricted not to leave $B(e, 2 \sigma)$. Now, $Q$ is radial: the transition probability between two vertices depends only on their distance in $\mathbf{T}$. Hence, if $k \in \mathbf{N}$ and $x \in C_{i}=$ $\{y \in \mathbf{T} \mid d(e, y)=i\}, i \leq 2 \sigma$, then we may write

$$
f_{\sigma, \tau}(i)=f(i)=\operatorname{Pr}\left[\exists n: Y_{n}^{*}=e \mid Y_{0}^{*}=x\right]
$$

We shall have $\sigma=\sigma(\rho), \tau=\tau(\rho)$, where $\rho=r\left(x_{i_{j}}\right) \rightarrow \infty$. Therefore, we now study the asymptotic behaviour of $f_{\sigma, \tau}(i)$ as $\sigma \rightarrow \infty, \sigma \leq \tau$ and $i / \sigma$ tends to a finite limit $(i \leq 2 \sigma)$. We introduce further notation: for $i, j \in[\sigma]=$ $\{1,2, \ldots, \sigma\}$ and $x \in C_{\sigma+i}$, define

$$
\begin{array}{r}
\mathrm{a}(i, j)=\operatorname{Pr}\left[\text { The first visit of } Y_{n}^{*} \text { to } B(e, \sigma)\right. \\
\text { occurs at some } \left.y \in C_{j} \mid Y_{0}^{*}=x\right] .
\end{array}
$$

Then $\mathrm{a}(i, j)$ does not depend on the particular choice of $x$. Besides

$$
\mathscr{A}=(\mathrm{a}(i, j))_{i, j \in[\sigma]},
$$


we introduce four other $\sigma \times \sigma$-matrices $\mathscr{P}=(p(i, j)), \mathscr{Q}=(q(i, j)), \mathscr{R}=$ $(r(i, j)), \mathscr{S}=(s(i, j))$, where for $i, j \in[\sigma]$,

$$
\mathrm{p}(i, j)=\operatorname{Pr}\left[Y_{n+1}^{*} \in C_{j} \mid Y_{n}^{*}=x \in C_{\sigma+i}\right]= \begin{cases}q^{\lfloor(j-i) / 2\rfloor} / b(\tau), & \text { if } i \leq j, \\ 0, & \text { otherwise }\end{cases}
$$

$$
\mathrm{q}(i, j)=\operatorname{Pr}\left[Y_{n+1}^{*} \in C_{\sigma+j} \mid Y_{n}^{*}=x \in C_{\sigma+i}\right]=q^{\lfloor(\sigma+j-i) / 2\rfloor} / b(\tau) ;
$$

$$
\mathrm{r}(i, j)=\operatorname{Pr}\left[Y_{n+1}^{*} \in C_{j} \mid Y_{n}^{*}=x \in C_{i}\right]= \begin{cases}(q+1) q^{j-1} / b(\tau), & \text { if } i+j \leq \sigma, \\ \mathrm{q}(i, j), & \text { otherwise }\end{cases}
$$

$$
\mathrm{s}(i, j)=\operatorname{Pr}\left[Y_{n+1}^{*} \in C_{\sigma+j} \mid Y_{n}^{*}=x \in C_{i}\right]= \begin{cases}q^{\lfloor(2 \sigma+j-i) / 2\rfloor} / b(\tau), & \text { if } i \geq j, \\ 0, & \text { otherwise }\end{cases}
$$

Finally, we introduce the column vectors

$$
\mathbf{f}=(f(i))_{i \in[\sigma]}, \quad \mathbf{g}=(f(\sigma+i))_{i \in[\sigma]} \quad \text { and } \quad \mathbf{b}=(1 / b(\tau))_{i \in[\sigma]} .
$$

The following lemma is proved by simple stopping time arguments, factoring through the first step of the walk.

Lemma 5. (a) $\mathscr{A}=\mathscr{P}+\mathscr{Q} \mathscr{A}$,

(b) $\mathbf{f}=\mathbf{b}+\mathscr{R} \mathbf{f}+\mathscr{S} \mathbf{g}$,

(c) $\mathbf{g}=\mathscr{A} \mathbf{f}$.

For the sake of simplicity, we assume from now on that

$$
\sigma \text { is even. }
$$

With some effort, one calculates $\mathscr{A}$ from Lemma 5(a): set

$$
\alpha=\frac{\sigma q^{\sigma / 2}}{2 b(\tau)}, \quad \beta=\alpha q^{-1 / 2} \quad \text { and } \quad \gamma=\frac{1}{(1-\alpha)^{2}-\beta^{2}} \cdot \frac{2}{\sigma b(\tau)} .
$$

Then

$$
\mathrm{a}(i, j)= \begin{cases}q^{(j-i) / 2} \gamma\left((1-\alpha)\lceil j / 2\rceil+q^{-1 / 2} \beta\lfloor j / 2\rfloor\right), & \text { if } i-j \text { is even } \\ q^{(j-i) / 2} \gamma\left(\beta\lceil j / 2\rceil+q^{-1 / 2}(1-\alpha)\lfloor j / 2\rfloor\right), & \text { if } i-j \text { is odd }\end{cases}
$$

We can now proceed to give an asymptotic estimate of $\mathbf{f}$ and $\mathbf{g}$. In the following calculations, 1 is the vector with constant entries one.

Proposition 3. If $\sigma \rightarrow \infty$ and $\tau \geq \sigma$, then uniformly for $i \in[\sigma]$,

$$
1+\mathscr{R} \mathbf{1}(i) \leq f(i) b(\tau) \leq 1+\mathscr{R} \mathbf{1}(i)+O\left(q^{3 \sigma / 4-\tau}\right)
$$

with

$$
\mathscr{R} \mathbf{1}(i)=q^{\sigma-\tau-i / 2} \xi_{i}\left(1+O\left(q^{-\tau}\right)\right)
$$


where $\left(q^{1 / 2}+1\right) /(q+1) \leq \xi_{i} \leq\left(q+q^{1 / 2}\right) /(q+1)$, and

$$
f(\sigma+i)=q^{\sigma / 2-2 \tau-i / 2} \eta_{p a r(i)}(1+o(1))
$$

where $\eta_{\text {par }(i)}>0$ depends on the parity of $i$.

Proof. All the following $O(\cdot)$ and $o(\cdot)$ will be uniform in $i \in[\sigma]$. The formulas for $\mathscr{R} \mathbf{1}(i)$ and $\xi_{i}$ are easily obtained from (5.4c). In particular,

$$
\mathscr{R} \mathbf{1}(i) \leq \mathscr{R} \mathbf{1}(1)=q^{\sigma-\tau} \frac{2}{q+1}\left(1+O\left(q^{-\tau}\right)\right)
$$

Furthermore, one obtains from (5.7) and (5.8) that

$$
\mathscr{A} \mathbf{1}(i)=O\left(\sigma q^{\sigma / 2-\tau-i / 2}\right)
$$

so that

$$
\mathscr{S} \mathscr{A} \mathbf{1}(i) \leq \frac{1}{b(\tau)} q^{\sigma-i / 2} \sum_{j=1}^{i} q^{j / 2} \mathscr{A} \mathbf{1}(j)=O\left(i \sigma q^{3 \sigma / 2-2 \tau-i / 2}\right)=q^{\sigma-\tau} o(1)
$$

Hence, if $\sigma$ is large enough, $\tau \geq \sigma$, then

$$
(\mathscr{R}+\mathscr{S} \mathscr{A}) \mathbf{1} \leq \frac{5}{2 q+2} \mathbf{1}
$$

Now, by Lemma 5 ,

$$
\mathbf{f}=\mathbf{b}+\mathscr{R} \mathbf{f}+\mathscr{S} \mathscr{A} \mathbf{f}
$$

If we set $\mathbf{f}_{1}=\mathbf{b}, \mathbf{f}_{k+1}=\mathbf{b}+\mathscr{R} \mathbf{f}_{k}+\mathscr{S} \mathscr{A} \mathbf{f}_{k}$, then the $\mathbf{f}_{k}$ tend to $\mathbf{f}$ monotonically from below, and (5.10) together with an inductive argument yields $\mathbf{f}_{k} \leq$ $(2 q+2) \mathbf{b} /(2 q-3)$ for all $k$, so that

$$
\mathbf{b} \leq \mathbf{f} \leq \frac{2 q+2}{2 q-3} \mathbf{b}
$$

In particular, $\mathbf{f} \geq \mathbf{b}+\mathscr{R} \mathbf{b}$, which proves the lower bound of $f(i) b(\tau)$ in (a). If we combine (5.9) and (5.12), then (5.11) gives

$$
f(i) b(\tau)=1+b(\tau) \mathscr{R} \mathbf{f}(i)+O\left(i \sigma q^{3 \sigma / 2-2 \tau-i / 2}\right)
$$


By applying (5.11) once again, and by making use again of (5.12) and of the asymptotic formula for $\mathscr{R} \mathbf{1}(i)$, it follows that

$$
\begin{aligned}
b(\tau) \mathscr{R} \mathbf{f}(i) \leq & \sum_{j \leq \sigma / 2} \mathrm{r}(i, j) \frac{2 q+2}{2 q-3} \\
& +\sum_{j>\sigma / 2} \mathrm{r}(i, j)\left(1+b(\tau) \mathscr{R} \mathbf{f}(j)+O\left(j \sigma q^{3 \sigma / 2-2 \tau-j / 2}\right)\right) \\
\leq & \frac{2 q+2}{2 q-3} \sum_{j \leq \sigma / 2} \mathrm{r}(1, j)+\mathscr{R} \mathbf{1}(i) \\
& +\sum_{j>\sigma / 2} \mathrm{r}(i, j)\left(O\left(q^{\sigma-\tau-j / 2}\right)+O\left(j \sigma q^{3 \sigma / 2-2 \tau-j / 2}\right)\right) \\
= & O\left(q^{\sigma / 2-\tau}\right)+\mathscr{R} \mathbf{1}(i)+\sum_{j>\sigma / 2} \mathrm{r}(i, j) O\left(q^{3 \sigma / 4-\tau}\right) \\
= & O\left(q^{\sigma / 2-\tau}\right)+\mathscr{R} \mathbf{1}(i)+O\left(q^{\sigma-\tau-i / 2}\right) O\left(q^{3 \sigma / 4-\tau}\right) \\
= & \mathscr{R} \mathbf{1}(i)+O\left(q^{3 \sigma / 4-\tau}\right) .
\end{aligned}
$$

This completes the proof of (a). To obtain (b), one uses Lemma 5(c) and combines (5.8) with (a) in a straightforward, but lengthy calculation. We omit the details.

We now come back to the setting of the end of $\S 4$, assuming that in addition to $(4.1)$,

$$
\Phi(n) \leq C \log _{q} n \text { for all } n \geq n_{0},
$$

where $C>0$. Choose $R$ as in (4.2) and set $L=3 r(e)+R+1$. We intend to verify the condition of Lemma 4 . To this end, choose

$$
i_{1}=n_{0}, \quad i_{j+1}=i_{j}+\left\lfloor 3 C \log _{q} i_{j}\right\rfloor+L .
$$

We shall need the following observation.

\section{Lemma 6.}

$$
\lim _{j \rightarrow \infty} 3 C \frac{j \log _{q} j}{i_{j}}=1 .
$$

The proof is obtained by further lengthy, but basic calculations; see the appendix.

Proof of the Theorem. Set $r_{j}=r\left(x_{i_{j}}\right)$. If $\left\{r_{j}\right\}$ has a bounded subsequence, then the sum in Lemma 4 diverges. Therefore we assume that $r_{j} \rightarrow \infty$. If $y \in B\left(x_{i_{j}}, 3 r_{j}+R\right)$ then $\sigma_{j} \leq r(y) \leq \tau_{j}$, where

$$
\sigma_{j}=r_{j}-\left\lfloor C \log _{q}\left(3 r_{j}+R\right)\right\rfloor \text { and } \tau_{j}=r_{j}+\left\lfloor C \log _{q}\left(3 r_{j}+R\right)\right\rfloor \text {. }
$$

We may assume that $2 \sigma_{j} \geq \tau_{j}$ and that $\sigma_{j}$ is even for all $j$ (otherwise, we replace $\sigma_{j}$ by $\left.\sigma_{j}-1\right)$. Then, if $v \in V_{i_{j}}$ we have

$$
V_{i_{j}} \subseteq B\left(v, 2 \sigma_{j}\right) \subseteq B\left(x_{i_{j}}, 3 r_{j}+R\right)
$$


The latter set does not intersect $V_{i_{j+1}}$, since

$$
r_{j} \leq r(e)+C \log _{q} i_{j} .
$$

In $B\left(v, 2 \sigma_{j}\right)$, the entries of $P_{r}$ are minorized by those of $Q_{\sigma_{j}, \tau_{j}}$ as defined in (5.1). Therefore,

$$
F_{V_{i_{j+1}}}(u, v) \geq f_{\sigma_{j}, \tau_{j}}(d(u, v)) \quad \text { for all } u, v \in V_{i_{j}} .
$$

Note that $\operatorname{diam}_{\mathbf{T}}\left(V_{i_{j}}\right) \leq \theta_{j}=r_{j}+R+\left\lfloor C \log _{q}\left(r_{j}+R\right)\right\rfloor$. If $j$ is sufficiently large then $\theta_{j} \leq 2 \sigma_{j}$, and we see from Proposition 3 that

$$
f_{\sigma_{j}, \tau_{j}}(d(u, v)) \geq \eta q^{\sigma_{j}-2 \tau_{j}-\theta_{j} / 2},
$$

if $0<\eta<\min \left\{\eta_{0}, \eta_{1}\right\} \quad\left(\eta_{0}, \eta_{1}\right.$ as in Proposition $\left.3 \mathrm{~b}\right)$. The exponent of $q$ in (5.16) is larger than $\kappa\left(r_{j}\right)$, where

$$
\kappa(\rho)=-\frac{3}{2} \rho-\frac{7}{2} C \log _{q}(3 \rho+R)-\frac{1}{2} R .
$$

Now (5.15) yields

$$
q^{\kappa\left(r_{j}\right)} \geq i_{j}^{-3 C / 2}\left(3 C \log _{q}\left(i_{j}+L-1\right)\right)^{-7 C / 2} q^{-(L-1) / 2},
$$

and by Lemma $6, \sum_{j} q^{\kappa\left(r_{j}\right)}$ diverges whenever $C<2 / 3$.

\section{FinAL REMARKS}

A. Our Theorem should be considered as a "prototype" of a result which allows several directions of extension and generalization. On an arbitrary (locally finite, connected) graph, the equivalent of Lemma 1 is not true in general. Infinite graphs where it does remain true are usually called "radial" or "distanceregular", see [Iv] for their classification: they are made up in a tree-like way from infinitely many copies of the finite complete graph $K_{m}(m \geq 2)$, such that exactly $k$ copies of $K_{m}$ meet at each vertex $(k \geq 2)$. For $\mathbf{T}, m=2$ and $k=q+1$. The generalization of our Theorem to all distance-regular graphs (with $k m>4$ in order to have nonconstants in $\mathscr{H}^{+}$) is an obvious exercise.

As mentioned in $\S 1$, our Theorem is of "hyperbolic" type, whereas the result in the continuous setting is "Euclidean". One could ask for the "correct" Euclidean analogue of the MVP on $\mathbf{T}$.

B. In our approach, we have tried to emphasize the connection between the MVP and the underlying graph structures, and in particular the relation between Martin boundaries and the space of ends. A different approach, which offers rather general tools for the study of the MVP and its converse, is "mass cancellation" following the lines of [He, Ba2]. The verification of the resulting conditions (e.g . in our setting of a Lipschitz-type assumption, (1.5) and (5.13)) appears to require similar efforts as the way of proof we have chosen.

C. We return to the setting of $\S 5$. If we drop the restriction that $Y_{n}^{*}$ does not leave $B(e, 2 \sigma)$, that is, if we replace all $Y_{k}^{*}$ by $Y_{k}$ in (5.2), (5.3) and (5.4), 
then the matrices $\mathscr{P}, \mathscr{Q}, \mathscr{R}$ and $\mathscr{S}$ remain the same, and in Lemma 5 , the only change occurs in (a), where we get

$$
\mathscr{A}=\mathscr{P}+\mathscr{Q} \mathscr{A}+\mathscr{S}^{2} \text {. }
$$

This makes the entries of $\mathscr{A}, \mathbf{f}$ and $\mathbf{g}$ larger, but in the asymptotic analysis, Proposition 3 still remains true for the unrestricted $\left\{Y_{n}\right\}$.

One could interpret this in the following way: If $r_{j}$ is large enough, then $\operatorname{diam}_{\Gamma_{\tau}}\left(V_{i_{j}}\right)=2$ by (4.4) and (5.13), so that the maximal number of steps needed to reach $v$ from $u$ for $u, v$ in $V_{i_{j}}$ is 2 . However, in the tree, $d(u, v) \leq$ $r_{j}+R+\Phi\left(r_{j}+R\right)$ is only very slightly larger than $\sigma_{j}$; in the limit, the "maximal number of steps" is $3 / 2$ instead of 2 .

This reasoning also suggests that it will be rather difficult to improve the bound $2 / 3$ (which is the inverse of the above "maximal number of steps") within the present approach.

D. We now exhibit a radius function $r$ on $\mathbf{T}$ and a bounded function on $\mathbf{T}$ which has the corresponding MVP but fails to be harmonic.

Example. Define

$$
r(e)=2, \quad r(x)=3 \cdot 2^{\left\lceil\log _{2} d(x, e)\right\rceil} \text { if } x \neq e .
$$

Set $U_{0}=\{e\}, U_{k}=\left\{x \in \mathbf{T} \mid\left\lceil\log _{2} d(x, e)\right\rceil=k-1\right\}$ for $k \geq 1$, and define a function $h$ on $\mathbf{T}$ by

$$
h(x)=(-1)^{k} h_{k} \quad \text { for } x \in U_{k} \text {, }
$$

where $h_{k}, k \in \mathbf{N}_{0}$, are real numbers. If we set $r_{k}=r(x), x \in U_{k}$, and $s_{k}=2^{k}$, then some calculations show that $h \in \mathscr{H}_{r}$ if and only if

$$
\begin{gathered}
q(q+1) h_{2}=(q+1)^{2} h_{0}+(q+1) h_{1} \text { and, for } k \geq 0, \\
\left(b\left(r_{k+1}\right)-b\left(s_{k+1}\right)\right) h_{k+3}=b\left(r_{k}\right) h_{k}+b\left(r_{k+1}\right) h_{k+1}+\left(b\left(s_{k+1}\right)-b\left(r_{k}\right)\right) h_{k+2} .
\end{gathered}
$$

If we set $h_{0}=0, h_{1}=1$ then it is not hard to see that (6.4) determines a bounded sequence of positive numbers, and the resulting bounded function $h$ on $\mathbf{T}$ has the MVP with respect to $r$. However, $h$ is radial: $h(x)$ depends only on $d(e, x)$, and the only radial bounded harmonic functions on $\mathbf{T}$ are the constants, so that $h$ is not harmonic. Observe that $r(x) \leq 6 d(x, e)$ for $x \neq e$, but $r$ does not satisfy a Lipschitz-type condition (1.5), as it has unbounded jumps.

E. On the other hand, the condition of Lemma 4 is only sufficient. Under assumption (1.5), the two Martin boundaries might even coincide if $\Gamma_{r}$ has only one end, which is the case for rapidly growing $\Phi$. We have not succeeded to compute any counterexample for this case. Note that in the continuous setting of the result of Veech stated in $\S 1$, there also seems to be a certain lack of efficient counterexamples (besides the obvious one of [Ve1], see [Ve2, Conjecture 1.2]). We do not have any conjecture concerning the existence of counterexamples where (1.5) does hold. 
Question. Is there a radius function $r: \mathbf{T} \rightarrow \mathbf{N}$ satisfying a condition of type (1.5), such that $\mathscr{H}_{r}^{+} \backslash \mathscr{H}^{+}$is nonvoid?

\section{APPENDIX}

Proof of Lemma 6. By a change of the base, we can get rid of the factor $3 C$. Hence, we assume that

$$
i_{1} \in \mathbf{N}, \quad i_{j+1}=i_{j}+\left\lfloor\log _{\theta} i_{j}\right\rfloor+L,
$$

where $\theta>1$ and $L \in \mathbf{N}$. From some $k_{0}$ onward, we can define $m_{k}=$ $\max \left\{j \mid i_{j}<\theta^{k}\right\}$ and $n_{k}=m_{k}+1$. We have:

$$
\begin{aligned}
& \text { If } n_{k}<j \leq n_{k+1}, \text { then } i_{j}=i_{n_{k}}+\left(j-n_{k}\right)(k+L), \\
& \theta^{k}-(k+L-1) \leq i_{m_{k}}<\theta^{k} \leq i_{n_{k}}<\theta^{k}+(k+L-1) .
\end{aligned}
$$

Hence, we get

$$
m_{k+1}-m_{k}=n_{k+1}-n_{k}=\left(i_{n_{k+1}}-i_{n_{k}}\right) /(k+L),
$$

and, by (A.1),

$$
\frac{\theta^{k+1}-\theta^{k}-(k+L-1)}{k+L}<m_{k+1}-m_{k}<\frac{\theta^{k+1}-\theta^{k}+k+L}{k+L} .
$$

We obtain

$$
\begin{aligned}
m_{k} & <\sum_{l=k_{0}}^{k-1} \frac{\theta^{l+1}-\theta^{l}+l+L}{l+L}+m_{k_{0}} \leq(\theta-1) \sum_{l=0}^{k-1} \frac{\theta^{l}}{l+L}+k-k_{0}+m_{k_{0}}, \\
m_{k} & >\sum_{l=k_{0}}^{k-1} \frac{\theta^{l+1}-\theta^{l}-(l+L-1)}{l+L}+m_{k_{0}} \\
& \geq(\theta-1) \sum_{l=0}^{k-1} \frac{\theta^{l}}{l+L}-k+k_{0}+m_{k_{0}}-(\theta-1) \sum_{l=0}^{k_{0}} \frac{\theta^{l}}{l+L} .
\end{aligned}
$$

The combination of these inequalities yields

$$
\left|m_{k}-(\theta-1) \sum_{l=0}^{k-1} \frac{\theta^{l}}{l+L}\right|<k+K \text { for every } k \geq k_{0},
$$

where $K$ is a constant. Now consider

$$
a_{k}=\sum_{l=0}^{k-1} \frac{\theta^{l-k}}{l+L} \quad \text { and } \quad A(z)=\sum_{k=1}^{\infty} a_{k} z^{k}, \quad z \text { complex }
$$

One computes

$$
A(z)=\frac{1}{z^{L-1}(\theta-z)}\left(\log (1-z)-\sum_{k=1}^{L-1} \frac{z^{k}}{k}\right) .
$$


The radius of convergence of the power series in (A.4) is one, and the only singularity of $A(z)$ with $|z|=1$ is $z=1$. By the logarithmic version of Darboux's theorem (see e.g. Chapter 8 in F. W. J. Olver: Asymptotics and special functions, Academic Press, New York, 1974), the $a_{k}$ are asymptotically equivalent with the coefficients of $\frac{1}{\theta-1} \log (1-z)$, developed at $z=0$. Hence, $a_{k} \sim 1 /(\theta-1) k$ as $k \rightarrow \infty$. Together with (A.3), we get

$$
\lim _{k \rightarrow \infty} \frac{k m_{k}}{\theta^{k}}=1 \text { and } \lim _{k \rightarrow \infty} \frac{k m_{k}}{i_{m_{k}}}=1 .
$$

On the other hand, $k-1 \leq \log _{\theta} m_{k}<k$, and

$$
\lim _{k \rightarrow \infty} \frac{m_{k} \log _{\theta} m_{k}}{i_{m_{k}}}=\lim _{k \rightarrow \infty} \frac{n_{k} \log _{\theta} n_{k}}{i_{n_{k}}}=1 .
$$

In the second limit, we have used that $i_{j+1} / i_{j} \rightarrow 1$ as $j \rightarrow \infty$.

For $j \in \mathbf{N}$, let $k(j)$ be such that $n_{k(j)}<j \leq n_{k(j)+1}$. Choose $\varepsilon>0, k_{\varepsilon} \geq k_{0}$ such that $(1-\varepsilon) n_{k} \log _{\theta} n_{k}<i_{n_{k}}<(1+\varepsilon) n_{k} \log _{\theta} n_{k}$ and $k+L<(1+\varepsilon)\left(k+\log _{\theta} e\right)$ for all $k \geq \varepsilon$. Suppose $j \geq n_{k_{\varepsilon}}$. By (A.1), the point $\left(j, i_{j}\right)$ lies on the line which connects $\left(n_{k(j)}, i_{\left.n_{k(j)}\right)}\right)$ and $\left(n_{k(j)+1}, i_{n_{k \mid j+1}}\right)$, and by convexity of $x \mapsto$ $(1-\varepsilon) x \log _{\theta} x$, it lies above the point $\left(j,(1-\varepsilon) j \log _{\theta} j\right)$ :

$$
(1-\varepsilon) j \log _{\theta} j<i_{j} \quad \text { for all } j \geq n_{k_{\varepsilon}} .
$$

On the other hand, consider $f_{\varepsilon}(x)=(1+\varepsilon) x \log _{\theta} x$. We have

$$
f_{\varepsilon}^{\prime}\left(n_{k(j)}\right) \geq(1+\varepsilon)\left(k(j)+\log _{\theta} e\right) \text {. }
$$

We have seen that $\left(j, i_{j}\right)$ lies on the line with slope $k(j)+L$ issuing from $\left(n_{k(j)}, i_{n_{k(j)}}\right)$. On the right of the latter point, the graph of $f_{\varepsilon}(x)$ lies above this line by our choice of $\varepsilon,\left(\right.$ A.7) and the convexity of $f_{\varepsilon}(x)$. In particular, $\left(j, f_{\varepsilon}(j)\right)$ lies above $\left(j, i_{j}\right)$ :

$$
i_{j}<(1+\varepsilon) j \log _{\theta} j \text { for all } j \geq n_{k_{\varepsilon}} .
$$

Together with (A.6), (A.8) completes the proof.

\section{REFERENCES}

[Ba1] J. R. Baxter, Restricted mean values and harmonic functions, Trans. Amer. Math. Soc. 167 (1972), 451-463.

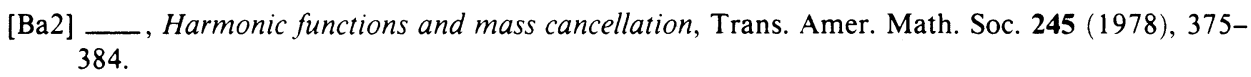

[Ca1] P. Cartier, Fonctions harmoniques sur un arbre, Symposia Math. 9 (1972), 203-270.

[Ca2] __ Harmonic analysis on trees, Proc. Sympos. Pure Math., vol. 26, Amer. Math. Soc., Providence, R.I., 1972, pp. 419-424.

[De] Y. Derriennic, Marche aléatoire sur le groupe libre et frontière de Martin, Z. Wahrsch. Verw. Gebiete 32 (1975), 261-276.

[Do] J. Dodziuk, Difference equations, isoperimetric inequality and transience of certain random walks, Trans. Amer. Math. Soc. 284 (1984), 787-794. 
[DK] J. Dodziuk and W. S. Kendall. Combinatorial Laplacians and isoperimetric inequality, From Local Times to Global Geometry, K. D. Ellworthy, ed., Pitman Res. Notes Math. Ser. 150, 1986, pp. 68-74.

[DM] E. B. Dynkin and M. B. Malyutov, Random walks on groups with a finite number of generators, Soviet Math. Dokl. 2 (1961), 399-402.

[Fe] W. Feller, Boundaries induced by nonnegative matrices, Trans. Amer. Math. Soc. 83 (1956), 19-54.

[FP] A. Figà-Talamanca and M. A. Picardello, Harmonic analysis on free groups, Lecture Notes in Pure and Appl. Math., vol. 87, Dekker, New York and Basel, 1987.

[Ge] P. Gerl, Random walks on graphs with a strong isoperimetric property, J. Theoret. Probab. 1 (1988), 171-187.

[He] D. Heath, Functions possessing restricted mean value properties, Proc. Amer. Math. Soc. 41 (1973), 588-595.

[Iv] A. A. Ivanov, Bounding the diameter of a distance-regular graph, Soviet Math. Dokl. 28 (1983), 149-152.

[Ke] O. D. Kellogg, Converses of Gauss' theorem on the arithmetic mean, Trans. Amer. Math. Soc. 36 (1934), 227-242.

[KSK] J. G. Kemeny, J. L. Snell and A. W. Knapp, Denumerable Markov chains, 2nd ed., Springer, New York, Heidelberg and Berlin, 1976.

[Mo] B. Mohar, Isoperimetric inequalities, growth and the spectrum of graphs, Simon Fraser Univ., 1987, preprint.

[PW1] M. A. Picardello and W. Woess, Martin boundaries of random walks: ends of trees and groups, Trans. Amer. Math. Soc. 302 (1987), 185-205.

[PW2] _ _ Harmonic functions and ends of graphs, Proc. Edinburgh Math. Soc. (in print).

[Ve1] W. Veech, A zero-one law for a class of random walks and a converse to Gauss' mean value theorem, Ann. of Math. 97 (1973), 189-216.

[Ve2] __ A converse to the mean value theorem for harmonic functions, Amer. J. Math. 97 (1975), $1007-1027$.

Dipartimento di Matematica, Università dell'Aquila, 67100 L'Aquila, Italy (Current address of M. A. Picardello)

Institüt für Mathematik und ANgewandte Geometrie, Montanuniversität, A-8700 LEOBEN, Austria

Current address (Wolfgang Woess): Dipartimento di Matematica, Università di Milano, via C. Saldini, 50, 20133 Milano, Italy 